\title{
Evaluación de la densidad de cultivo sobre el crecimiento y supervivencia de larvas de chita Anisotremus scapularis (Tschudi, 1846) en laboratorio
}

\author{
Angélica Castro Fuentes", Noemi Cota, Melissa Montes y lili Carrera \\ Instituto del Mar del Perú (IMARPE), Esquina Gamarra y Gral. Valle, s/n, PO Box 22, Chucuito, Perú. \\ ORCID Angélica Castro Fuentes (D) https://orcid.org/0000-0002-6616-5436, Noemi Cota (D) https://orcid.org/0000-0001-8944-8159, \\ Melissa Montes (D) https://orcid.org/0000-0002-6149-8709, Lili Carrera (D) https://orcid.org/0000-0002-1570-142X
}

Marine and

Fishery Sciences

MAFIS

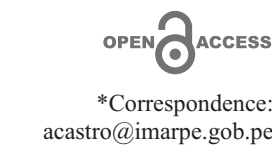

Received: 19 May 2021 Accepted: 14 August 2021

ISSN 2683-7595 (print) ISSN 2683-7951 (online)

https://ojs.inidep.edu.ar

Journal of the Instituto Nacional de Investigación y Desarrollo Pesquero (INIDEP)

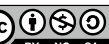

This work is licensed under a Creative Commons AttributionNonCommercial-ShareAlike 4.0 International License
RESUMEN. La larvicultura de peces marinos en condiciones de laboratorio está influenciada por factores como la densidad de cultivo, la nutrición, la calidad del agua, que afectan directamente el crecimiento y la supervivencia. Este estudio tuvo como objetivo determinar el efecto de la densidad de cultivo sobre el crecimiento, la supervivencia y el factor de condición de las larvas de chita Anisotremus scapularis en condiciones de laboratorio. Las larvas se obtuvieron a partir del desove natural de reproductores mantenidos en condiciones controladas. Se evaluaron cuatro densidades de cultivo 5, 10, 15 y 20 larvas $\mathrm{l}^{-1}$. El ensayo se llevó a cabo durante $60 \mathrm{~d}$ a $19{ }^{\circ} \mathrm{C}$ en un sistema estático compuesto por 12 tanques con una capacidad de 1001 cada uno. Los resultados no mostraron diferencias significativas en las tasas de crecimiento específico, pero sí en la supervivencia y el factor de condición (K). La densidad de cultivo de 10 larvas $1^{-1}$ obtuvo el valor más alto de $\mathrm{K}(2,14 \pm 0,31)$ durante el experimento, por lo que se sugiere la misma como densidad de cultivo inicial de chita.

Palabras clave: Cultivo larval, crecimiento, densidad larval, supervivencia, Perú.

Assessment of stocking density on growth and survival of Peruvian grunt Anisotremus scapularis (Tschudi, 1846) larvae in laboratory

ABSTRACT. Marine fish larviculture under laboratory condition is influenced by factors such as stocking density, nutrition, water quality, which affect directly growth and survival. This study was aimed to determine the effect of stocking density on growth, survival and condition factor of Peruvian grunt Anisotremus scapularis larvae reared in laboratory conditions. Fish larvae were obtained by natural spawning of broodstock keep under controlled conditions. Four culture densities of 5, 10, 15 and 20 larvae $1^{-1}$ were tested. Larvae rearing were carried out for $60 \mathrm{~d}$ at $19{ }^{\circ} \mathrm{C}$ in a static system consisting of 12 tanks with a capacity of 1001 each. Results showed significant differences in the condition factor $(\mathrm{K})$ and survival, but no such differences were detected in the specific growth rate. Stocking density of 10 larvae $1^{-1}$ obtained the highest $\mathrm{K}$ value $(2.14 \pm 0.31)$ during the experiment; therefore, this value is suggested as the initial stocking density for Peruvian grunt.

Key words: Growth performance, stocking density, survival, larval rearing, Peru. 


\section{INTRODUCCIÓN}

La chita Anisotremus scapularis (Tschudi, 1846) se distribuye desde Manta (Ecuador) hasta Antofagasta (Chile) Isla Coco y Galápagos (Chirichigno y Cornejo 2001). Es una especie bentopelágica y carnívora e importante en la interacción de las comunidades litorales marinas, ya que utiliza recursos tróficos tanto de ambientes arenosos como rocosos (Iannacone y Alvariño 2009). Pertenece a la Familia Haemulidae, estrechamente relacionada con las familias Lutjanidae (pargos) y Sparidae (sargos y doradas) (Orrell et al. 2002, 2004; Tavera et al. 2012). Es valorada por la calidad de su carne con un costo aproximado en los supermercados de US\$13,97 por kilo y en los terminales pesqueros de US\$ 8,57 el kilo, en donde se comercializa fresca (PRODUCE 2017). Los desembarques de esta especie han disminuido en los últimos años hasta llegar a $49 \mathrm{t}$ (PRODUCE 2020). En este sentido, el Laboratorio de Cultivo de Peces del Instituto del Mar del Perú (IMARPE) inició en 2013 las investigaciones sobre el acondicionamiento de esta especie, obteniéndose el ciclo de vida en cautiverio (IMARPE 2015), el acondicionamiento y manejo de reproductores en cautiverio (Carrera et al. 2018), la descripción del desarrollo embrionario (Montes et al. 2019) y el establecimiento de un protocolo de cultivo larval (Castro et al. 2021). No obstante estos primeros avances, el cultivo larval sigue siendo escasamente dominado.

Durante la larvicultura de peces marinos en condiciones controladas, existen muchos factores que influyen sobre el crecimiento y supervivencia (Houde 1975; Tucker 1998; Lazo 2000; CiveraCerecedo et al. 2004). Uno de ellos es la densidad de siembra de huevos y/o larvas que pueden afectar la calidad del agua, limitar el espacio y aumentar la mortalidad (Sakakura y Tsukamoto 2002; Szkudlarek y Zakés 2007; Abdo de la Parra et al. 2010). Se han realizado diversos estudios de densidad de siembra con especies similares a la chita, como en Lutjanus guttatus con 20 huevos $1^{-1}$ (Boza-Abarca et al. 2008), 10 larvas $1^{-1}$ en $L$. argentimaculatu (Leu et al. 2003), 8,6 larvas 1-1 en L. analis (Watanabe et al. 1998), 30 huevos $1^{-1}$ en L. guttatus (Abdo de la Parra et al. 2010) y 20 huevos $1^{-1}$ en Dentex dentex (Koumoundouros et al. 2000), en los cuales se obtuvieron altas tasas de crecimiento. Por otro lado, el estudio de la condición nutricional o factor de condición permite evaluar el estado fisiológico de las larvas, que es reflejo de las condiciones de cultivo a las que estuvieron expuestas (Diaz y Pájaro 2013). Hasta la fecha no existen reportes sobre la densidad de siembra para el cultivo larvario de la chita, por lo que el objetivo del presente trabajo es conocer los efectos de la densidad de cultivo en el crecimiento, supervivencia y factor de condición en larvas de chita $A$. scapularis en condiciones de laboratorio.

\section{MATERIALES Y MÉTODOS}

\section{Mantenimiento de reproductores y desove}

Los reproductores de chita A. scapularis provinieron del medio natural, fueron acondicionados en dos tanques de fibra de vidrio de $2,5 \mathrm{~m}^{3}$ a una capacidad efectiva de $1,9 \mathrm{~m}^{3}$, conectados a un sistema de recirculación de agua de mar (Carrera et al. 2018) en el Laboratorio de Cultivo de Peces del IMARPE. Los reproductores fueron mantenidos con un foto y termoperiodo natural según los datos registrados por la NOAA (National Oceanic and Atmospheric Administration) para la zona de Callao (Perú), obteniendo el desove cuando se simularon los meses de primavera-verano. La calidad del agua se monitoreó día por medio con un multiparámetro portátil (YSIPro1020®) registrando la temperatura $\left(18,83 \pm 0,20{ }^{\circ} \mathrm{C}\right), \mathrm{pH}(7,79 \pm$ $0,17)$, oxígeno disuelto $\left(7,71 \pm 0,44 \mathrm{mg} \mathrm{l}^{-1}\right)$ y porcentaje de saturación $(82,66 \pm 4,22 \%)$, mientras 
que los productos nitrogenados como amonio total $\left(0,04 \pm 0,03 \mathrm{mg} \mathrm{L}^{-1}\right)$, nitrito $\left(0,76 \pm 0,33 \mathrm{mg} \mathrm{L}^{-1}\right)$ y nitrato $\left(26,04 \pm 8,80 \mathrm{mg} \mathrm{L}^{-1}\right)$, se registraron en forma semanal. Los reproductores se alimentaron con trozos de anchoveta Engraulis ringens cuatro veces a la semana a una tasa de alimentación entre 4 y $5 \%$ de la biomasa total de cada tanque.

Los desoves ocurrieron de forma espontánea en los tanques. Los huevos flotantes se colectaron utilizando una malla de $500 \mu \mathrm{m}$ y se colocaron en un balde de 201 con agua de mar filtrada y esterilizada por luz ultravioleta (UV) por $30 \mathrm{~min}$, para permitir la decantación de los huevos no viables. Se contabilizaron volumétricamente 25.520 huevos viables (flotantes) que fueron colocados en un tanque de incubación de 7001 de volumen total llenado al 50\% con agua de mar estéril (UV) en sistema estático. La aireación estuvo provista por una piedra difusora, el fotoperiodo se ajustó a $12 \mathrm{~h}$ luz : $12 \mathrm{~h}$ oscuridad (12 HL : $12 \mathrm{HO}$ ) y la temperatura a $19{ }^{\circ} \mathrm{C}$ hasta su eclosión a las $48 \mathrm{~h}$ (Castro et al. 2021). Las larvas se mantuvieron en ese tanque hasta la siembra en las unidades experimentales.

\section{Cultivo larvario}

Se utilizaron 15.000 larvas $(0,32 \pm 0,01 \mathrm{~cm}$ de longitud total y $0,002 \pm 0,001 \mathrm{~g}$ de peso seco) provenientes de un solo desove. Las larvas fueron trasladadas el día 1 después de su eclosión (DDE) a 12 tanques de fibra de vidrio $(0,60 \mathrm{~m}$ diámetro y 0,50 m altura) con un volumen efectivo de 1001 . Se utilizó agua de mar (35 PSU) esterilizada por UV. Los tanques fueron provistos de aireación constante por medio de piedras difusoras y la iluminación se realizó con luz blanca entre 915 y 1.100 lux. Durante los 10 primeros días de cultivo se utilizó un fotoperiodo $24 \mathrm{HL}: 00 \mathrm{HO}$ y luego se cambió a 12 HL : 12 HO hasta el final del experimento. La temperatura de la sala se mantuvo constante mediante un sistema de aire acondicionado. Diariamente se registraron la temperatura $\left(19,9 \pm 0,71{ }^{\circ} \mathrm{C}\right), \mathrm{pH}(8,58 \pm 0,37)$, oxígeno disuelto $\left(7,33 \pm 0,46 \mathrm{mg} \mathrm{l}^{-1}\right)$ y porcentaje de satu- ración $(80,53 \pm 4,68 \%)$, los cuales se mantuvieron en el rango del cultivo de larvas reportado para la Familia Sparidae (Elbal et al. 2004).

El cultivo larval se llevó a cabo en sistemas estáticos (sin flujo de agua), iniciando con un volumen de 601 de agua de mar estéril y agregando 101 por día por los próximos cinco días de cultivo. A los 6 DDE se bajó el nivel a 601 con un sifón y una malla de $100 \mu \mathrm{m}$ y se procedió de manera similar a lo descrito anteriormente hasta los 10 DDE. Posteriormente, se realizaron recambios de agua del 10 a $100 \%$ diario con entrada y salida de agua hasta el final de la experiencia (Tabla 1). Se utilizó la técnica de "agua verde" (Skiftesvik et al. 2003; Sanaye et al. 2014; Hu et al. 2018; Wang et al. 2019), adicionando a los tanques de cultivo las microalgas Isochrysis galbana y Nanochloropsis oculata a una concentración de $8 \times 10^{6}$ células $\mathrm{ml}^{-1}$ y $6 \times$ $10^{7}$ células $\mathrm{ml}^{-1}$, respectivamente. La alimentación de las larvas se inició el día 2 DDE al no detectar la presencia del saco vitelino. Para ello, se utilizaron rotíferos Brachionus plicatilis hasta el 20 DDE, y luego se realizó el cambio de alimento a Artemia hasta el $36 \mathrm{DDE}$, tiempo en que se inicia la coalimentación o deshabituación del alimento vivo al inerte (destete) con un alimento microparticulado comercial (Otohime ${ }^{\circledR}$ ), hasta ser suministrado en su totalidad hasta final del experimento (60 DDE). Los alimentos vivos utilizados durante el experimento fueron enriquecidos con Selco Expreso ${ }^{\circledR}$ según las indicaciones del proveedor. La densidad del alimento vivo se controló tres veces al día siguiendo el protocolo de alimentación del laboratorio (Castro et al. 2021) (Tabla 1).

\section{Diseño experimental}

Se evaluaron cuatro densidades de cultivo: D5 (5 larvas $\left.1^{-1}\right)$, D10 (10 larvas 1-1), D15 (15 larvas $\left.1^{-1}\right)$ y $20\left(20\right.$ larvas $\left.1^{-1}\right)$ con tres repeticiones cada una. Para las determinaciones morfométricas al inicio y al final del experimento, las mues- 
Tabla 1. Protocolo del cultivo larval de chita Anisotremus scapularis en condiciones de laboratorio. Iso: Isochrysis galbana $(8 \times$ $10^{6}$ células $\left.\mathrm{ml}^{-1}\right)$. Np: Nanochloropsis oculata $\left(6 \times 10^{7}\right.$ células $\left.\mathrm{ml}^{-1}\right)$.

Table 1. Larviculture protocol of Peruvian grunt (Anisotremus scapularis) under laboratory conditions. Iso: Isochrysis galbana

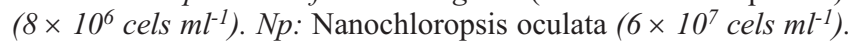

\begin{tabular}{|c|c|c|c|c|c|c|}
\hline $\begin{array}{l}\text { Días después } \\
\text { de la eclosión }\end{array}$ & $\begin{array}{l}\text { Rotíferos } \\
\quad\left(\mathrm{ml}^{-1}\right)\end{array}$ & $\begin{array}{l}\text { Artemia } \\
\left(\mathrm{ml}^{-1}\right)\end{array}$ & $\begin{array}{c}\text { Alimento } \\
\text { micro-particulado }\end{array}$ & $\begin{array}{l}\text { Recambio de } \\
\text { agua }\left(\% \mathrm{~d}^{-1}\right)\end{array}$ & $\begin{array}{c}\text { Volumen de } \\
\text { agua efectiva (l) }\end{array}$ & $\begin{array}{l}\text { Microalga } \\
\text { Iso-Np (1) }\end{array}$ \\
\hline 0 & & & 0 & 60 & $0,4-1,6$ & \\
\hline 2 & 1 & & & 0 & 70 & $0,4-1,6$ \\
\hline 3 & 1 & & & 0 & 80 & $0,2-0,8$ \\
\hline 4 & 1 & & & 0 & 90 & $0,4-1,6$ \\
\hline 5 & 2 & & & 0 & 100 & $0,2-0,8$ \\
\hline 6 & 2 & & & 50 & 60 & $0,8-3,2$ \\
\hline 7 & 2 & & & 0 & 70 & $0,8-3,2$ \\
\hline 8 & 2 & & & 0 & 80 & $0,8-3,2$ \\
\hline 9 & 2 & & & 0 & 90 & $0,4-1,6$ \\
\hline 10 & 3 & & & 0 & 100 & $0,4-1,6$ \\
\hline 11 & 3 & & & 10 & 100 & $0,8-3,2$ \\
\hline $12-14$ & 3 & & & 20 & 100 & $1,6-6,4$ \\
\hline $15-16$ & 4 & & & 30 & 100 & $1,6-6,4$ \\
\hline $17-20$ & 4 & 0,5 & & 40 & 100 & $1,6-6,4$ \\
\hline $21-25$ & 3 & 1,0 & & 70 & 100 & $0,8-3,2$ \\
\hline $26-30$ & 0 & 2,0 & & 100 & 100 & $0,4-1,6$ \\
\hline $31-35$ & 0 & 3,0 & & 100 & 100 & $0,2-0,8$ \\
\hline $36-40$ & 0 & 2,0 & Ad libitum & 100 & 100 & $0,2-0,8$ \\
\hline $41-45$ & 0 & 1,0 & Ad libitum & 100 & 100 & $0,2-0,8$ \\
\hline $46-60$ & 0 & 0,0 & Ad libitum & 100 & 100 & 0 \\
\hline
\end{tabular}

tras de larvas se anestesiaron en una solución de MS-222 a una concentración de $250 \mathrm{mg}^{-1}$ (AVMA, 2014). El peso seco (g) se estimó siguiendo la metodología de Pepín (1995), y la longitud total $(\mathrm{cm})$ se determinó en un microscopio Leica DM1000 LED con el software de análisis de imágenes LAS versión 4.3. La supervivencia se evaluó al final y se calculó mediante la fórmula:

Supervivencia $(\%)=$

$$
\frac{\text { Larvas al final del experimento }}{\text { Larvas al inicio del experimento }} \times 100
$$

Se calculó la tasa de crecimiento específico (TCE) en relación a la longitud y peso de cada tratamiento de densidad (De Oliveira et al. 2012; Lugert et al. 2014) mediante:

$\operatorname{TCE}(\%)=100 \times \frac{\left(\ln W_{t f}-\ln W_{t i}\right)}{\mathrm{t}}$

donde TCE es la tasa de crecimiento específico $\left(\% \mathrm{~g} \mathrm{~d}^{-1} \mathrm{y}_{\%} \mathrm{~cm} \mathrm{~d} \mathrm{~cm}^{-1}\right)$; ln es el logaritmo natural; $W_{t f}$ es la longitud/peso final y $W_{t i}$ es la longitud/peso inicial.

El estado de condición por tratamiento se estimó mediante el índice de Fulton (K) (Ricker 1975): 
$\mathrm{K}=100 \times \frac{\mathrm{W}}{\mathrm{L}^{3}}$

donde:

W: peso final $(\mathrm{g})$;

L: longitud final $(\mathrm{cm})$.

\section{Análisis estadístico}

El análisis estadístico fue realizado con el programa estadístico R (R Core Team 2019). Las variables longitud, peso, TCE, K y supervivencia de los diferentes tratamientos fueron comparados estadísticamente con un análisis de varianza de una vía (ANOVA), previo análisis de los supuestos de normalidad y homocedasticidad de varianzas con el test de Shapiro Wilk y Bartlett, respectivamente. Si las variables no cumplieran estos supuestos, se aplicó la prueba no paramétrica de Kruskal-Wallis. Cuando se observaron diferencias significativas, se aplicó la prueba post hoc de Tukey en el caso de las variables como prueba paramétrica y de Mann Whitney como prueba no paramétrica. Los valores expresados en porcentaje se transformaron en arcosen de su raíz cuadrada antes del ANOVA. El nivel de significancia establecido para todos los análisis fue de 0,05.

\section{RESULTADOS}

En relación al peso y longitud no se encontraron diferencias significativas $(\mathrm{p}>0,05)$ entre los tratamientos (Tabla 2; Figura 1). La TCE en longitud $\left(\% \mathrm{~cm} \mathrm{~d}^{-1}\right)$ no presentó diferencias significativas entre los tratamientos $(\mathrm{p}>0,05)$, mientras que la TCE en peso $\left(\% \mathrm{~g} \mathrm{~d}^{-1}\right)$ presentó diferencias significativas $(\mathrm{p}<0,05)$, siendo el tratamiento D20 significativamente diferente a los otros tratamientos (Figura 2).

La supervivencia fue significativamente diferente entre los tratamientos $(\mathrm{p}<0,05)$. La mayor supervivencia se obtuvo con los tratamientos D5 y D10 siendo significativamente diferente $(\mathrm{p}<0,05)$

Tabla 2. Parámetros biológicos de larvas de chita Anisotremus scapularis a diferentes densidades de cultivo (promedio \pm desviación estándar; $\mathrm{n}=3$ ). DDE: días después de la eclosión. Diferentes letras indican diferencias significativas $(\mathrm{p}<0,05)$.

Table 2. Biological parameters of Peruvian grunt (Anisotremus scapularis) larvae at different culture densities (mean \pm standard desviation; $n=3)$. DDE: days after hatching. Different letters indicate significant differences $(p<0.05)$.

Densidad de cultivo (larvas $\left.1^{-1}\right)$

\begin{tabular}{llll}
\hline D5 & D10 & D15
\end{tabular}

\begin{tabular}{lcccc} 
Peso $(\mathrm{g})$ & & & \\
$5 \mathrm{DDE}$ & $0,003 \pm 0,001$ & $0,001 \pm 0,001$ & $0,004 \pm 0,002$ & $0,001 \pm 0,000$ \\
$60 \mathrm{DDE}$ & $0,30 \pm 0,120$ & $0,32 \pm 0,148$ & $0,29 \pm 0,132$ & $0,27 \pm 0,102$ \\
& $(\mathrm{n}=30)$ & $(\mathrm{n}=30)$ & $(\mathrm{n}=30)$ & $(\mathrm{n}=30)$ \\
Longitud $(\mathrm{cm})$ & & & & \\
$5 \mathrm{DDE}$ & $0,31 \pm 0,010$ & $0,33 \pm 0,003$ & $0,31 \pm 0,016$ & $0,32 \pm 0,003$ \\
$60 \mathrm{DDE}$ & $2,48 \pm 0,278$ & $2,43 \pm 0,474$ & $2,42 \pm 0,474$ & $2,60 \pm 0,579$ \\
& $(\mathrm{n}=30)$ & $(\mathrm{n}=30)$ & $(\mathrm{n}=30)$ & $(\mathrm{n}=30)$ \\
Supervivencia $(\%)$ & $24,58 \pm 17,58^{\mathrm{a}}$ & $12,54 \pm 2,47^{\mathrm{ab}}$ & $7,69 \pm 1,64^{\mathrm{b}}$ & $5,52 \pm 1,27^{\mathrm{b}}$ \\
$K$ & $1,85 \pm 0,44^{\mathrm{a}}$ & $2,14 \pm 0,62^{\mathrm{a}}$ & $1,91 \pm 0,39^{\mathrm{a}}$ & $1,64 \pm 0,68^{\mathrm{b}}$ \\
\hline
\end{tabular}




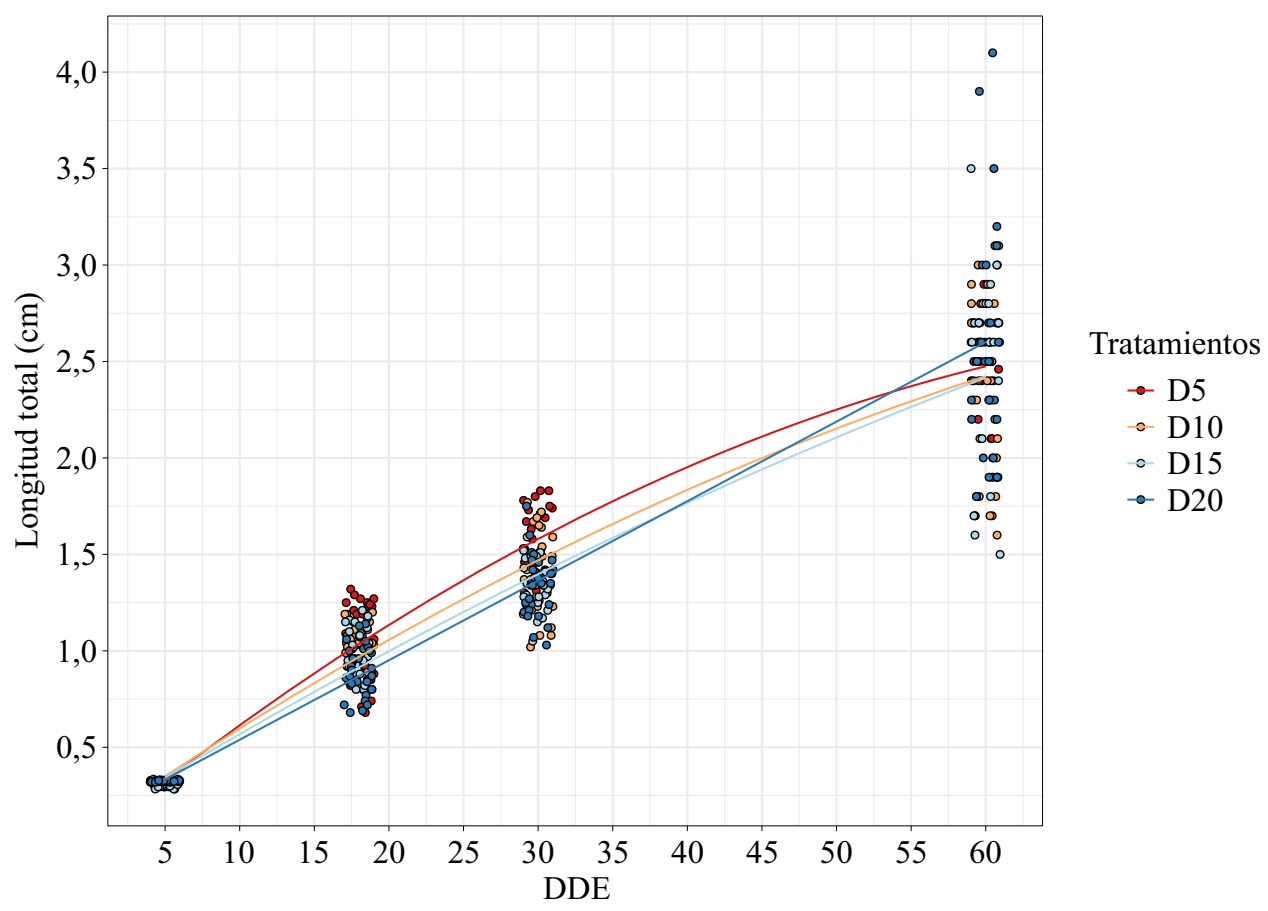

Figura 1. Crecimiento en longitud (L, cm) de larvas de chita Anisotremus scapularis a diferentes densidades de cultivo. DDE: días después de la eclosión.

Figure 1. Growth in length (L, cm) of Anisotremus scapularis larvae at different culture densities. DDE: days after hatching.

a los tratamientos D15 y D20 (Tabla 2). El factor de condición también presentó diferencias significativas entre los tratamientos $(\mathrm{p}<0,05)$. El tratamiento que obtuvo el más alto $\mathrm{K}$ fue el D10; no obstante, no se detectaron diferencias significativas con el D5 y D15, pero sí con el tratamiento D20 (Tabla 2).

\section{DISCUSIÓN}

La densidad de siembra en los peces marinos puede afectar el crecimiento, supervivencia, factor de condición y tasa de crecimiento (Houde 1975; Tucker 1998; Montero et al. 1999). Asimismo, se relaciona a la densidad como uno de los factores determinantes en la larvicultura, porque también afecta las interacciones sociales como agresividad, jerarquía y canibalismo, lo cual da como resultado variaciones en el tamaño, supervivencia y rendimiento del crecimiento en las poblaciones de peces (Hatziathanasiou et al. 2002). La escala del cultivo (laboratorio o comercial) puede verse también afectada por la densidad de siembra larval (Álvarez-González et al. 2001; Conides y Glamuzina 2001; Hitzfelderg et al. 2006; Benetti et al. 2008; Castro et al. 2019).

En el presente trabajo, las diferentes densidades de siembra larval afectaron el crecimiento, supervivencia y el factor de condición. En tal sentido, de las cuatro densidades evaluadas en el cultivo de chita en condiciones de laboratorio, D5 registró la mayor supervivencia, aunque sin observarse diferencias significativas con D10. Aunque no se cuenta con reportes de supervivencia en esta especie, en espáridos como L. guttatus a densidades de 27, 16 y 9 larvas $1^{-1}$ se obtuvo una supervivencia de $1,44,1,45$ y $2,82 \%$, respectivamente, a los 45 DDE (Abdo de la Parra et al. 

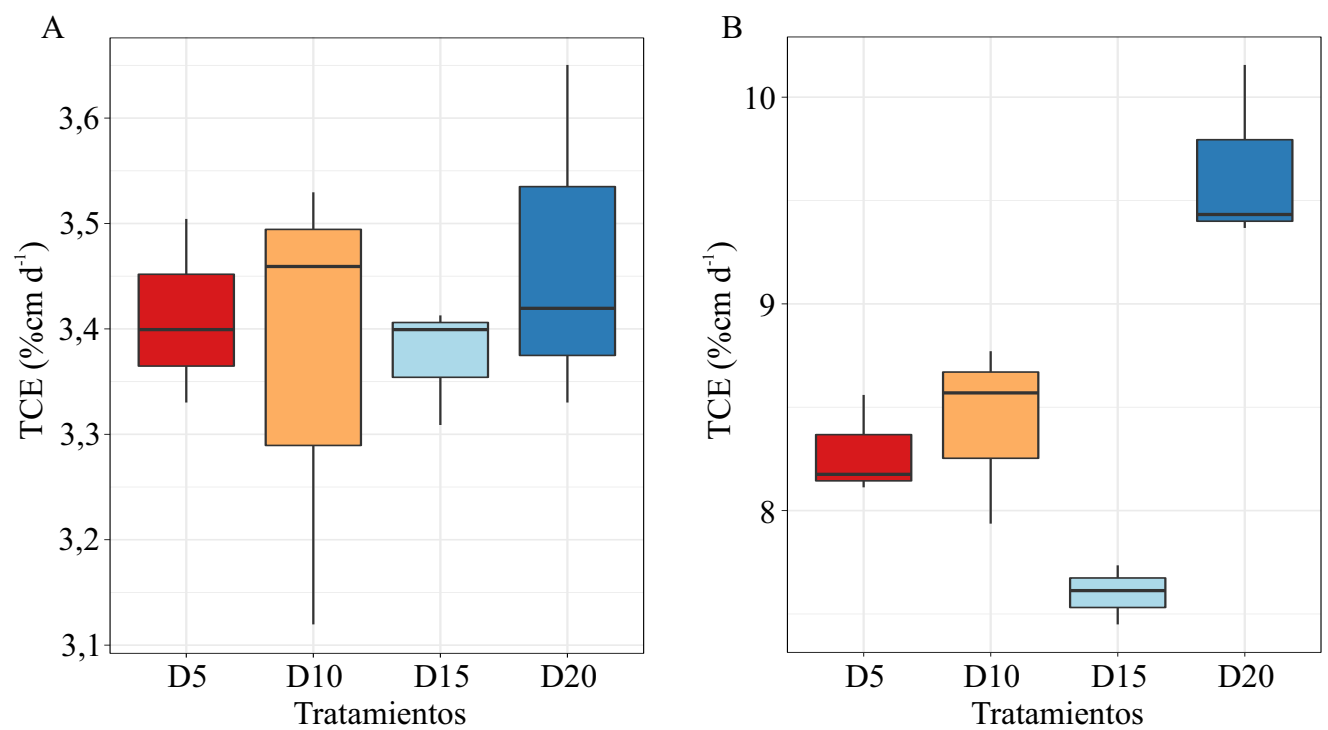

Figura 2. Tasas de crecimiento especifico (TCE) en longitud (A) y peso (B) en el cultivo de larvas de chita Anisotremus scapularis en diferentes densidades de cultivo.

Figure 2. Specific growth rates (TCE) in length (A) and weight (B) in the culture of Anisotremus scapularis larvae at different culture densities.

2010). En larvas de Acanthopagrus zatus a densidades de 17 larvas $1^{-1}$ se obtuvo una supervivencia de $14,6 \%$ a los 50 DDE (Leu y Chou 1996). En otras especies como Lates calcarifer, la supervivencia fue del $28 \%$ a una densidad de 20 larvas $1^{-}$ ${ }^{1}$ a los 20 DDE (Salama 2007). Estudios a mayor escala en larvas de Sparus aurata a densidad de 60 larvas $1^{-1}$ registraron una supervivencia de 39,31\% a los 15 DDE (Parra y Yúfera, 2000), mientras que Pousão-Ferrerra et al. (2003) con densidades de 58,9 larvas $1^{-1}$ a los 20 DDE obtuvieron una supervivencia de 19,4\%. En larvas de Argyrosomus regius con densidades de 50 y 100 larvas $1^{-1}$ registraron supervivencias de 40,92 y 53,24\%, respectivamente (Roo et al. 2010). En peces planos como Paralichthys lethostigma, densidades cultivo de 10, 20, 40 y 80 larvas $1^{-1}$ exhibieron supervivencias de 7,6, 6,7, 6,6 y 6,8\%, respectivamente, hasta el 21 DDE (Daniels et al. 1996); mientras que larvas de Solea aegyptiaca a $50,80,110$ y 140 larvas $1^{-1}$ obtuvieron una supervivencia de $7,55,8,53,10,60$ y $7,38 \%$, respectivamente, a los 36 DDE (Saleh et al. 2016).
Al final del presente ensayo (60 DDE) no se encontraron diferencias significativas en la longitud de los individuos entre los tratamientos, registrándose el valor más alto alcanzado por $A$. $s c a$ pularis en el tratamiento de D20 $(2,60 \pm 0,579$ $\mathrm{cm}$ ), el cual fue menor a las alcanzadas por $S t i-$ zostedion vitreurn $(4,70 \pm 0,02 \mathrm{~cm})$ con la misma densidad a los 45 DDE (Fox y Flowers 1990), Acanthopagrus zatus $(2,00 \pm 1,50 \mathrm{~cm})$ a densidad de 17 larvas $1^{-1}$ a los 50 DDE (Leu y Chou 1996), y Lates calcarifer $(0,76 \mathrm{~cm})$ a densidad de 20 larvas $1^{-1}$ a los 20 DDE (Salama 2007).

En relación al incremento en peso, en el tratamiento D10 se obtuvo un promedio de $0,32 \pm 0,14$ $\mathrm{g}$ pero no se encontraron diferencias significativas entre los otros tratamientos, por lo tanto, no hubo influencia de la densidad en el peso de larva. Sin embargo, en otros trabajos se observaron valores más altos a lo reportado en menor tiempo, en los cuales el peso no presentó diferencias significativas en relación a la densidad, como en $L$. guttatus que fue 1,35 $\pm 0,95 \mathrm{~g}$ a 30 huevos $1^{-1}$ (Abdo de la Parra et al. 2010) al 46 DDE; en $S$. vitreurn se 
obtuvo un peso de $0,71 \pm 0,83 \mathrm{~g}$ al $45 \mathrm{DDE}$ a 20 larvas $1^{-1}$ (Fox y Flowers 1990) y en Dicentrarchus labrax se obtuvo 6,21 $\pm 0,02 \mathrm{~g}$ al $22 \mathrm{DDE}$ a 50 larvas $1^{-1}$ (Hatziathanasiou et al. 2002).

Se sabe que las interacciones sociales y la jerarquía de dominancia conducen a la supresión de la ingesta de alimentos y limitan el crecimiento en individuos subordinados (Montero et al. 2009). Las larvas de mayor tamaño, tienden a consumir más alimento, lo cual conduce a una tasa de crecimiento muy alta. Alternativamente, las larvas más pequeñas consumen menos alimento y pueden participar en maniobras evasivas para evitar a los peces dominantes, lo que resulta en alto gasto de energía y crecimiento reducido (Xie et al. 2011). En tal sentido, la tasa de crecimiento específico se ve afectada por la disponibilidad de alimento y el tamaño de la larva, como se evidenció en Pseudosciaena crocea, la TCE $\left(\% \mathrm{~d}^{-1}\right)$ fue de $8,2 \pm 0,1$ a 42 DDE (Xie et al. 2011); en larvas de $S$. aurata a $60 \mathrm{DDE}$ con un fotoperiodo de $12 \mathrm{~h}$ luz y sin flujo de agua, la TCE fue de 9,7 $\pm 3,78$ (Tandler y Helps 1985); en Amphiprion clarkii, la TCE fue de $6,38 \pm 0,4$ a $23{ }^{\circ} \mathrm{C}$ al concluir la metamorfosis, aproximadamente al 30 DDE (Ye et al. 2011). En el presente trabajo, hubo diferencias en la TCE en peso $\left(\% \mathrm{~g} \mathrm{~d}^{-1}\right)$, siendo el tratamiento D20 $(9,65 \pm$ $0,43)$ el que alcanzó el mayor incremento durante el experimento. En D. labrax, la TCE $\left(\% \mathrm{~g} \mathrm{~d}^{-1}\right)$ fue de 3,72 $\pm 0,52$ a una densidad de 20 larvas $1^{-1}$ (Hatziathanasiou et al. 2002). En larvas de $S$. aurata a densidades de 5 larvas $1^{-1}$ la TCE fue de 0,01 a los 39 DDE (Soltan et al. 2015), mientras que Eid et al. (2018) con densidades de 25 larvas de $S$. aurata $\mathrm{L}^{-1}$ obtuvo una TCE de 0,04 a los 60 DDE. En cultivos masivos se ha obtenido una TCE de 0,01 en larvas de Diplodus sargus a den-

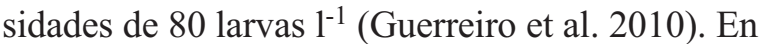
relación a la TCE en longitud $\left(\% \mathrm{~cm} \mathrm{~d}^{-1}\right)$ no presentaron diferencias entre los tratamientos, pero estudios en espáridos con larvas de L. guttatus se obtuvo un valor de 0,024 a los 26 DDE (BozaAbarca et al. 2008), menor a la densidad de 20 larvas $1^{-1}$ encontrado en este experimento.
El factor de condición es un indicador de las condiciones nutricionales (Blackwell et al. 2000; Cara et al. 2003; Froese 2006) a las que fueron sometidas las larvas durante el ensayo, obteniendo el tratamiento $\mathrm{D} 10$ el valor más alto de $\mathrm{K}=2,14 \pm$ 0,62 ; sin embargo, no se han registrado estudios previos del factor de condición en larvas de esta especie. Al respecto, en larvas de $S$. vitreurn a una densidad inicial de 20, 40 y 60 larvas $1^{-1}$ el valor de $\mathrm{K}$ fue de 0,63, 0,63 y 0,65, respectivamente (Fox y Flowers 1990). En larvas de Lithognathus lithognathus se obtuvo un valor de K de 1,19; en larvas de Pagrus pagrus se obtuvo un valor de K de 0,01 a los 35 DDE con una densidad de 20 larvas $1^{-1}$ (Andrade et al. 2012); en altas densidades con larvas de $S$. aurata (80 larvas $1^{-1}$ ) el valor de $\mathrm{K}$ fue 0,03 a los 50 DDE (Faria et al. 2011).

En general, la menor densidad larval en cultivo está asociada con un mayor crecimiento en la mayoría de las especies como A. regius, $S$. aurata, P. pagrus, D. dentex, Rachycentron canadum, $\mathrm{y}$ con parámetros de cultivo como la disponibilidad de alimentos y el espacio vital (Giménez y Estévez 2008; Roo et al. 2010), mientras que la alta densidad larval se asocia a tasas de crecimiento más bajas principalmente debido a la reducción del apetito, mayor comportamiento agresivo, baja calidad del agua o aumento de la competencia alimentaria (Roo et al. 2010). Por lo tanto, la densidad larval de peces se encuentra asociada a varios factores del cultivo, y sobre la base de los resultados obtenidos en el presente estudio en relación al factor de condición, se recomienda que el cultivo larvario de la chita $A$. scapularis sea realizado a una densidad inicial de 10 larvas $1^{-1}$ en condiciones de laboratorio.

\section{AGRADECIMIENTOS}

El presente trabajo de investigación fue financiado por el programa presupuestal $\mathrm{N}^{\circ} 0094$ Ordenamiento y Desarrollo de la Acuicultura, con 
el proyecto “Acondicionamiento y Reproducción de especies priorizadas chita Anisotremus scapularis" del Instituto del Mar del Perú.

\section{REFERENCIAS}

Abdo de la Parra MI, Rodríguez-Ibarra LE, Campillo-Martínez F, Velasco-Blanco G, García-Aguilar N, Álvarez-LaJonchÈre L, VolTOLINA D. 2010. Effect of stocking density on survival and growth of larval spotted rose snapper Lutjanus guttatus larvae. Rev Biol Mar Oceanogr. 45 (1): 141-146.

Álvarez-GonzÁlez C, Ortíz-Galindo S, Dumas S, MartínezDomínguez E, HernándezCeballos D, Grayeb-del Alamo T, MorenoLegorreta M, Peña-Martínez R. 2001. Effect of stocking density on the growth and survival of spotted sand bass Paralabrax maculatofasciutus larvae in a closed recirculating system. J World Aquac Soc. 32 (1): 130-137.

Andrade CA, Nascimento F, Conceição LE, Linares F, Lacuisse M, Dinis MT. 2012. Red porgy, Pagrus pagrus, larvae performance and nutritional condition in response to different weaning regimes. J World Aquac Soc. 43 (3): 321-334.

[AVMA] American Veterinary Medical AssoCIATION. 2014. American Veterinary Medical Association guidelines on euthanasia (formerly: report of the AVMA Panel on Euthanasia). $39 \mathrm{p}$.

Benetti DD, Sardenberg B, Welch A, Hoenig R, ORHUN MR, ZINK I. 2008. Intensive larval husbandry and fingerling production of cobia Rachycentron canadum. Aquac. 281 (1-4): 2227.

Blackwell BG, Brown ML, Willis DW. 2000. Relative weight (Wr) status and current use in fisheries assessment and management. Rev Fish Sci. 8 (1): 1-44.

Boza-Abarca J, Calvo-Vargas C, Solis-Ortiz
N, Komen J. 2008. Desove inducido y crecimiento del pargo manchado Lutjanus guttatus, en la Estación de Biología Marina de Puntarenas, Costa Rica. Cienc Mar. 34 (2): 239-252.

Cara JB, Moyano FJ, Cárdenas S, FernándezDíAZ C, YÚFERA M. 2003. Assessment of digestive enzyme activities during larval development of white bream. J. Fish Biol. 63 (1): 48-58.

Carrera l, Cota N, Linares J, Castro A, Orihuela L, Silva E, Montes M. 2018. Manual para acondicionamiento y reproducción de chita Anisotremus scapularis. Inf Inst Mar Perú. 45 (2): 263-276.

Castro A, Cota N, Montes M, Carrera L. 2021. Protocolo del cultivo larvario de chita Anisotremus scapularis en condiciones de laboratorio. Inf Inst Mar Perú. 48 (1): 20-24.

Castro A, Montes M, Orihuela L, Linares J, Cota N, Carrera L, Toledo P, Lazo JP. 2019. Effect of stocking density on growth and survival of fine flounder Paralichthys adspersus (Steindachner, 1867) larvae. Lat Am J Aquat Res. 47 (1): 1-8.

Chirichigno FN, Cornejo UM. 2001. Catálogo comentado de los peces marinos del Perú. Callao: Instituto del Mar del Perú. 500 p.

Civera-Cerecedo R, Alvarez-González CA, MoyANO-LÓPEz FJ. 2004. Nutrición y alimentación de larvas de peces marinos. En: CRUZ SuÁrez LE, Ricque Marie D, Nieto López MG, Villarreal D, Scholz U, González M, editores. Avances en nutrición acuícola VII. Memorias del VII Simposium Internacional de Nutrición Acuícola. 16-19 Noviembre, 2004. Hermosillo, Sonora, México.

Conides A, Glamuzina B. 2001. Study on the early larval development and growth of the red porgy, Pagrus pagrus with emphasis on the mass mortalities observed during this phase. Sci Mar. 65 (3): 193-200.

Daniels HV, Berlinsky DL, Hodson RG, SulliVAN CV. 1996. Effects of stocking density, salinity, and light intensity on growth and sur- 
vival of southern flounder Paralichthys lethostigma larvae. J World Aquac Soc. 27 (2): 153-159.

De Oliveira EG, Pinheiro AB, De Oliveira VQ, Da Silva AR, De Moraes MG, Rocha IR, Rocha IB, De Sousa RR, Costa FF. 2012. Effects of stocking density on the performance of juvenile pirarucu (Arapaima gigas) in cages. Aquaculture. 370-371: 96-101.

Diaz MV, PÁJaro M. 2013. Estudio de la condición nutricional de larvas de la población bonaerense de anchoíta (Engraulis anchoita) en relación con las características hidrográficas del área de crianza. Rev Invest Desarr Pesq. 23: 107-123.

Eid AE, Ali BA, Elghamry AM, Salama F, ElNaby A, AsmaA S. 2018. Effects of replacement of live food with dry diet on growth and survival rate for seabream (Sparus Aurata) Larvae. Egyp J Nut F. 21 (2): 573-581.

Elbal MT, García Hernández MP, LOZANo MT, Agulleiro B. 2004. Development of the digestive tract of gilthead sea bream (Sparus aurata L.). Light and electron microscopic studies. Aquaculture. 234: 215-238.

Faria AM, Chícharo MA, Gonçalves EJ. 2011. Effects of starvation on swimming performance and body condition of pre-settlement Sparus aurata larvae. Aquat Biol. 12 (3): 281289.

Fox MG, Flowers DD. 1990. Effect of fish density on growth, survival, and food consumption by juvenile walleyes in rearing ponds. $T$ Am Fish Soc. 119 (1): 112-121.

Froese R. 2006. Cube law, condition factor and weight-length relationships: history, metaanalysis and recommendations. J Appl Ichthyol. 22 (4): 241-253.

GIMÉNEZ G, EstéveZ A. 2008. Effect of larval and prey density, prey dose and light conditions on first feeding common dentex (Dentex dentex L.) larvae. Aquac Res. 39: 77-84.

Guerreiro I, De Vareilles M, Pousão-Ferreira P, Rodrigues V, Dinis MT, Ribeiro L.
2010. Effect of age-at-weaning on digestive capacity of white seabream (Diplodus sargus). Aquaculture. 300 (1-4): 194-205.

Hatziathanasiou A, Paspatis M, Houbart M, Kestemont P, Stefanakis S, Kentouri M. 2002. Survival, growth, and feeding in early life stages of European sea bass (Dicentrarchus labrax) intensively cultured under different stocking densities. Aquaculture. 205: 89102.

Hitzfelderg GM, Holt J, Fox J, McKee D. 2006. The effect of rearing density on growth and survival of cobia, Rachycentron canadum, larvae in a closed recirculating aquaculture system. J World Aquac Soc. 37 (2): 204-209.

HoudE ED. 1975. Effects of stocking density and food density on survival. growth and yield of laboratory-reared larvae of sea bream Archosargus rhomboidalis (L.) (Sparidae). J Fish Biol. 7: 115-129.

Hu J, LiU Y, MA Z, QIN JG. 2018. Feeding and development of warm water marine fish larvae in early life. En: YufERA, M. editor. Emerging Issues in Fish Larvae Research. Cham: Springer. p. 275-296.

IANNACONE J, AlvariÑo L. 2009. Aspectos cuantitativos de la parasitofauna de Anisotremus scapularis (Tschudi) (Osteichthyes, Haemulidae) capturados por pesquería artesanal en Chorrillos, Lima, Perú. Rev. Ibero-Latinoam Parasitol. 68 (1): 56-64.

[IMARPE] Instituto del Mar del Perú. 2015. Ciclo de vida de la chita Anisotremus scapularis. Serie de Divulgación Científica. Vol. 1. 1 (1). $20 \mathrm{p}$.

Koumoundouros G, Divanach P, Kentouri M. 2000. Development of the skull in Dentex dentex (Osteichthyes: Sparidae). Mar Biol. 136 (1): 175-184.

LAzo J. 2000. Conocimiento actual y nuevas perspectivas en el desarrollo de dietas para larvas de peces marinos. En: CruZ-SuÁrez LE, Ricque-Marie D, TAPIA-SAlazar M, Olvera-Novoa MA, Civera-Cerecedo R, edito- 
res. Avances en nutrición acuícola V. Memorias del V Simposium Internacional de Nutrición Acuícola. 19-22 Noviembre, 2000. Mérida, Yucatán, México.

Leu MY, Chen IH, FANG LS. 2003. Natural spawning and rearing of mangrove red snapper, Lutjanus argentimaculatus, larvae in captivity. Isr J Aquac. 55: 22-30.

LEU MY, CHOU YH. 1996. Induced spawning and larval rearing of captive yellowfin porgy, Acanthopagrus latus (Houttuyn). Aquaculture. 143 (2): 155-166.

Lugert V, Thaller G, Tetens J, Schulz C, KriETER J. 2014. A review on fish growth calculation: multiple functions in fish production and their specific application. Rev Aquac. 8: 30-42.

MONTERo D, IZQUIERdo MS, TORT L, RobaInA L, VERGARA JM. 1999. High stocking density produces crowding stress altering some physiological and biochemical parameters in gilthead seabream, Sparus aurata, juveniles. Fish Physiol Biochem. 20: 53-60.

Montero D, Lalumera G, Izquierdo MS, Caballero MJ, Saroglia M, Tort L. 2009. Establishment of dominance relationships in gilthead sea bream Sparus aurata juveniles during feeding: effects on feeding behaviour, feed utilization and fish health. J Fish Biol. 74 (4), 790-805.

Montes M, Castro AM, Linares JF, Orihuela LI, CARrera LJ. 2019. Embryonic development of Peruvian grunt Anisotremus scapularis (Perciformes: Haemulidae). Rev Biol Mar Oceanog. 54 (2): 166-173. doi:10.22370/ rbmo.2019.54.2.1881

Orrell TM, Carpenter KE, Musick JA, Graves JE. 2002. Phylogenetic and biogeographic analysis of the Sparidae (Perciformes: Percoidei) from cytochrome $b$ sequences. Copeia. 2002 (3): 618-631.

OrRell TM, CARPENTER KE. 2004. A phylogeny of the fish family Sparidae (porgies) inferred from mitochondrial sequence data. Mol Phylogenet Evol. 32 (2): 425-434.
Parra G, Yúfera M. 2000. Feeding, physiology and growth responses in first-feeding gilthead seabream (Sparus aurata L.) larvae in relation to prey density. J Exp Mar Biol Ecol. 243 (1): $1-15$.

PePíN P. 1995. An analysis of the length-weight relationship of larval fish: limitations of the general allometric model. Fish Bull. 93 (2): 419-426.

Pousão-Ferreira P, Santos P, Carvalho AP, Morais S, NARCiso L. 2003. Effect of an experimental microparticulate diet on the growth, survival and fatty acid profile of gilthead seabream (Sparus aurata L.) larvae. Aquacult Int. 11 (5): 491-504.

[PRODUCE] Ministerio DE LA PRODUCCión. 2017. Anuario estadístico pesquero y acuícola. [consultado 5 julio 2020]. http://ogeiee. produce.gob.pe/index.php/shortcode/oeedocumentos-publicaciones/publicacionesanuales/item/825-anuario-estadistico-pesquero-y-acuicola-2017.

[PRODUCE] Ministerio de LA Producción. 2020. Dato pesquero. [consultado 15 julio 2020]. https://www.produce.gob.pe/index.php/ shortcode/servicios-pesca/datero-pesquero.

R Core Team. 2019. R: A language and environment for statistical computing. R Foundation for Statistical Computing. Viena. https://www. R-project.org/.

RICKER WE. 1975. Computation and interpretation of biological statistics of populations. Bull Fish Res Board Can. 191: 382.

Roo J, Hernández CM, Borrero C, Schuchardt D, Fernández-Palacios H. 2010. Effect of larval density and feeding sequence on meagre (Argyrosomus regius; Asso, 1801) larval rearing. Aquaculture. 302: 82-88.

SAKAKURA Y, TsuKamoto K. 2002. Onset and development of aggressive behavior in the early life stage of Japanese flounder. Fish Sci. 68 (4): 854-861.

SAlama AJ. 2007. Effects of stocking density on fry survival and growth of Asian Sea Bass 
(Lates calcarifer). J King Abdulaziz Univ Mar Sci. 18: 53-61.

Saleh H, Mohammed R, Abou-Zied R, Allam S, AlJilany S. 2016. Impact of sex ratio and stocking density on the induced spawning of the Egyptian sole (Solea aegyptiaca; Chabanaud, 1927). Egyp J Aqua Biol Fish. 20 (1): 59-75.

Sanaye SV, Dhaker HS, Tibile RM, Mhatre VD. 2014. Effect of green water and mixed zooplankton growth and survival in neon tetra, Paracheirodon innesi (Myers, 1936) during larval and early fry rearing. World Academy of Science. Int J Biomed Eng Technol. 8 (2): 159-163.

Skiftesvik AB, Browman HI, St-Pierre JF. 2003. Life in green water: the effect of microalgae on the behavior of Atlantic cod (Gadus morhua) larvae. En: BROWMAN HI, SkifTESVIK AB, editores. The big fish bang. Proceedings of the 26th annual larval fish conference. Bergen: Inst Mar Res. p. 97-103.

Soltan MA, SHaker IM, AbD-Ella MM, SoliMAN ASM, DARWISH SI. 2015. Effect of early weaning on growth and survival rate of gilthead seabream (Sparus aurata) larvae. Proceeding of the second international Conference on Biotechnology Applications in Agriculture, Moshtohor and Hurghada, 8-11 April 2014. Faculty of Agriculture, Benha University. J Anim Sci Biotechnol (poultry and fish). 49-60.

SZKUDLAREK M, ZAKĘŚ Z. 2007. Effect of stocking density on survival and growth performance of pikeperch, Sander lucioperca (L.), larvae under controlled conditions. Aquacult
Inter. 15 (1): 67-81.

TAndler A, Helps S. 1985. The effects of photoperiod and water exchange rate on growth and survival of gilthead sea bream (Sparus aurata, Linnaeus; Sparidae) from hatching to metamorphosis in mass rearing systems. Aquaculture. 48 (1): 71-82.

tavera JJ, Acero A, Balart EF, Bernardi G. 2012. Molecular phylogeny of grunts (Teleostei, Haemulidae), with an emphasis on the ecology, evolution, and speciation history of New World species. BMC Evol Biol. 12 (1): 57.

TUCKER JW. 1998. The future of marine fish culture. En: TUCKER JW. editor. Marine fish culture. Boston: Springer. p. 587-588.

Wang J, Shu X, Wang WX. 2019. Micro-elemental retention in rotifers and their trophic transfer to marine fish larvae: influences of green algae enrichment. Aquaculture. 499: 374-380.

Watanabe W, Ellis E, Ellis S, Chaves J, ManFREDI C. 1998. Artificial propagation of mutton snapper Lutjanus analis, a new candidate marine fish species for aquaculture. J World Aquacult Soc. 29 (2): 176-187.

Xie F, Ai Q, Mai K, Xu W, Ma H. 2011. The optimal feeding frequency of large yellow croaker (Pseudosciaena crocea, Richardson) larvae. Aquac. 311 (1-4): 162-167.

Ye L, YANG SY, Zhu XM, LiU M, Lin JY, Wu KC. 2011. Effects of temperature on survival. development, growth and feeding of larvae of yellowtail clownfish Amphiprion clarkia (Pisces: Perciformes). Acta Ecol Sin. 31 (5): 241-245. 